CERN-TH/97-285

hep-th/9710163

\title{
CANONICAL EQUIVALENCE OF NON-ISOMETRIC $\sigma$-MODELS AND POISSON-LIE T-DUALITY
}

\author{
Konstadinos Sfetsos \\ Theory Division, CERN \\ CH-1211 Geneva 23, Switzerland
}

\begin{abstract}
We prove that a transformation, conjectured in our previous work, between phase-space variables in $\sigma$-models related by Poisson-Lie T-duality is indeed a canonical one. We do so by explicitly demonstrating the invariance of the classical Poisson brackets. This is the first example of a class of $\sigma$-models with no isometries related by canonical transformations. In addition we discuss generating functionals of canonical transformations in generally non-isometric, bosonic and supersymmetric $\sigma$-models and derive the complete set of conditions that determine them. We apply this general formalism to find the generating functional for Poisson-Lie T-duality. We also comment on the relevance of this work to D-brane physics and to quantum aspects of T-duality.
\end{abstract}

CERN-TH/97-285

October 1997

*e-mail address: sfetsos@mail.cern.ch 


\section{Introduction}

Among the different dualities in string theory T-duality [1] remains by far the best understood and, as such, it has influenced the development of the subject to a great extent. An elegant, as well as suitable for many applications, formulation of T-duality is as a canonical transformation on phase space. This was realized first for toroidal constant backgrounds [2]. The ultimate goal was and still is to extend the canonical equivalence for curved backgrounds, and eventually even to classify them.'t The first step in this direction was done for the non-Abelian dual of the $S U(2)$ Principal Chiral Model (PCM) with respect to the left (or right) action of $S U(2)$ [6] and for the duals of general backgrounds with respect to an Abelian isometry [7]. It was further realized that the canonical transformation for the $S U(2)$ PCM generates, with minor modifications, non-Abelian dualities for PCMs for any group [8] and, remarkably, even for the most general $\sigma$-model with a non-Abelian group of isometries acting from the left (or the right) only [9]. There is also formulation of non-Abelian duality in WZW models as a canonical transformation [9] (for some relevant remarks, see section 4). This has new interesting features since, unlike the previous cases, the duality group acts vectorially from the left and the right. The common feature of the works we have mentioned so far is that in order to implement the T-duality, either from the path integral point of view or via the canonical approach, a group of isometries was needed. In [10] the situation was improved by generalizing the concept of T-duality in the absence of isometries when the corresponding backgrounds fulfil certain conditions. This kind of T-duality, called Poisson-Lie T-duality, encompasses all Abelian and non-Abelian T-dualities we have mentioned, except that for WZW models. In [11] it was shown that $\sigma$-models related by Poisson-Lie T-duality may be seen as arising from a single action, thus hinting towards their canonical equivalence. However, the generating functional was obtained in an abstract form not suitable for extracting the explicit canonical map in phase space. Such a map was conjectured in 12 on the basis of symmetry arguments and correct limit in the case of the usual non-Abelian duality.

In this paper we are going to prove the canonical equivalence by explicitly demonstrating the invariance of the Poisson brackets under the so far conjectured transformation. Hence, we provide the first example of a class of $\sigma$-models that are, although not isometric, canonically equivalent and put Poisson-Lie T-duality on an equal footing with Abelian and non-Abelian duality, for which an explicit canonical formulation existed, as explained. In addition, we will systematically formulate the generating functional approach to canonical transformations for general bosonic and supersymmetric $\sigma$-models that do not necessarily have isometries. We will apply this general formalism to find perturbatively, in a sense to be explained in the text, the generating functional for PoissonLie T-duality. The initial motivation for the present work, besides the disentanglement of the existence of a canonical transformation on the existence of isometries, stems from

\footnotetext{
${ }^{1}$ Historically, the development of T-duality on curved backgrounds followed mainly the path integral approach. Besides the works we will explicitly mention, certain aspects of Abelian duality can be found in [3] and of non-Abelian duality in [4. For review articles, see [5].
} 
the fact that certain phenomena that present a problem from a field theoretical point of view are simply "gauge" artefacts from a string theoretical view point. This has been illustrated, by means of the interplay between world-sheet supersymmetry and Abelian 113] as well as non-Abelian T-duality [9], with the help of the canonical formulation; one would like to extend the discussion to Poisson-Lie T-duality (for work and discussion in this direction see [12]). The revival of open string theory sparked by [14, thanks to its relevance in understanding non-perturbative string dualities, brings up the interest in T-duality for open strings [15], in relation to D-branes [16]. Our results in this paper may help to understand backreaction effects of the D-branes due to a Fischler-Susskind type mechanism [17], as we explain in section 4 .

The organization of this paper is as follows: In section 2 we prove the canonical transformation for Poisson-Lie T-duality by showing the invariance of the classical Poisson brackets. In section 3 we discuss generating functionals in general bosonic and $N=1$ supersymmetric $\sigma$-models and apply for Poisson-Lie T-duality. In section 4 we conclude with an extensive discussion of possible future directions of this work. We have also written in appendix A the derivation of some non-trivial identities used in the proofs of section 2, and in appendix B the derivation of a manifestly Poisson-Lie T-duality invariant affine algebra.

\section{The canonical transformation}

\subsection{A brief review}

We consider a 2-dimensional non-linear $\sigma$-model for the $d$ field variables $X^{M}=\left(X^{\mu}, Y^{i}\right)$, where $X^{\mu}, \mu=1,2, \ldots, \operatorname{dim}(G)$ parametrize an element $g$ of a group $G$ and the rest are denoted by $Y^{i}, i=1,2, \ldots, d-\operatorname{dim}(G)$. We also introduce representation matrices $\left\{T_{a}\right\}$, with $a=1,2, \ldots, \operatorname{dim}(G)$ and the components of the left-invariant Maurer-Cartan forms $L_{ \pm}^{a}=L_{\mu}^{a} \partial_{ \pm} X^{\mu}$, with an inverse denoted by $L_{a}^{\mu}$. For notational convenience we will also use $L_{ \pm}^{i}=\partial_{ \pm} Y^{i}$ and $L_{ \pm}^{A}=\left(L_{ \pm}^{a}, L_{ \pm}^{i}\right)$. The light-cone coordinates on the world-sheet are $\sigma^{ \pm}=\frac{1}{2}(\tau \pm \sigma)$. The corresponding action has the form

$$
\begin{aligned}
S & =\frac{1}{2} \int L_{+}^{A} E_{A B}^{+} L_{-}^{B} \\
& =\frac{1}{2} \int L_{+}^{a} E_{a b}^{+} L_{-}^{b}+\partial_{+} Y^{i} \Phi_{i a}^{+} L_{-}^{a}+L_{+}^{a} \Phi_{a i}^{+} \partial_{-} Y^{i}+\partial_{+} Y^{i} \Phi_{i j} \partial_{-} Y^{j}
\end{aligned}
$$

Similarly we consider another $\sigma$-model for the $d$ field variables $\tilde{X}^{M}=\left(\tilde{X}^{\mu}, Y^{i}\right)$, where $\tilde{X}^{\mu}, \mu=1,2, \ldots, \operatorname{dim}(G)$ parametrize a different group $\tilde{G}$, whose dimension is, however, equal to that of $G$. The rest of the variables are the same $Y^{i}$ 's used in (1). Accordingly we introduce a different set of representation matrices $\left\{\tilde{T}^{a}\right\}$, with $a=1,2, \ldots, \operatorname{dim}(G)$ and the corresponding components of the left-invariant Maurer-Cartan forms $\tilde{L}_{ \pm a}=\tilde{L}_{\mu a} \partial_{ \pm} \tilde{X}^{\mu}$ 
(with inverse $\left.\tilde{L}^{\mu a}\right)$ as well as $\tilde{L}_{ \pm A}=\left(\tilde{L}_{ \pm a}, L_{ \pm}^{i}\right)$. The corresponding action has the form

$$
\begin{aligned}
\tilde{S} & =\frac{1}{2} \int \tilde{L}_{+A} \tilde{E}^{+A B} \tilde{L}_{-B} \\
& =\frac{1}{2} \int \tilde{L}_{+a} \tilde{E}^{+a b} \tilde{L}_{-b}+\partial_{+} Y^{i} \tilde{\Phi}_{i}^{+a} \tilde{L}_{-a}+\tilde{L}_{+a} \tilde{\Phi}^{+a}{ }_{i} \partial_{-} Y^{i}+\partial_{+} Y^{i} \tilde{\Phi}_{i j} \partial_{-} Y^{j}
\end{aligned}
$$

The matrix $E_{A B}^{+}$(for later use we also define $E_{A B}^{-}=E_{B A}^{+}$) in (11) may depend on all variables $X^{\mu}$ and $Y^{i}$ and similarly for the matrix $\tilde{E}^{+A B}$ in the action (2). Hence, we do not require any isometry associated with the groups $G$ and $\tilde{G}$.

The $\sigma$-models (11) and (2) will be dual to each other in the sense of Poisson-Lie T-duality [10] if the associated algebras $\mathcal{G}$ and $\tilde{\mathcal{G}}$ form a pair of maximally isotropic subalgebras into which the Lie algebra $\mathcal{D}$ of a Lie group $D$ known as the Drinfeld double can be decomposed. This implies that besides the usual commutators

$$
\left[T_{a}, T_{b}\right]=i f_{a b}{ }^{c} T_{c}, \quad\left[\tilde{T}^{a}, \tilde{T}^{b}\right]=i \tilde{f}^{a b}{ }_{c} \tilde{T}^{c},
$$

for the algebras $\mathcal{G}$ and $\tilde{\mathcal{G}}$, there is also the "mixed" commutator to consider

$$
\left[T_{a}, \tilde{T}^{b}\right]=i \tilde{f}_{a}^{b c} T_{c}-i f_{a c}^{b} \tilde{T}^{c}
$$

There is also a bilinear invariant $\langle\cdot \mid \cdot\rangle$ with the various generators obeying

$$
\left\langle T_{a} \mid T_{b}\right\rangle=\left\langle\tilde{T}^{a} \mid \tilde{T}^{b}\right\rangle=0, \quad\left\langle T_{a} \mid \tilde{T}^{b}\right\rangle=\delta_{a}{ }^{b}
$$

For more mathematical details one should consult the literature (for instance [18]). There remains to relate $E_{A B}^{+}$in (1) to $\tilde{E}^{+A B}$ in (2). In order to do that it is convenient to define matrices $a(g), b(g)$ and $\Pi(g)$ as

$$
g^{-1} T_{a} g=a_{a}{ }^{b} T_{b}, \quad g^{-1} \tilde{T}^{a} g=b^{a b} T_{b}+\left(a^{-1}\right)_{b}{ }^{a} \tilde{T}^{b}, \quad \Pi^{a b}=b^{c a} a_{c}{ }^{b} .
$$

Consistency restricts them to obey

$$
a\left(g^{-1}\right)=a^{-1}(g), \quad b^{T}(g)=b\left(g^{-1}\right), \quad \Pi^{T}(g)=-\Pi(g) .
$$

Similarly we define matrices $\tilde{a}(\tilde{g}), \tilde{b}(\tilde{g})$ and $\tilde{\Pi}(\tilde{g})$ by just replacing the untilded symbols by tilded ones. Then the various couplings in the $\sigma$-model actions (11), (2) are restricted to be of the form [10, 19]:

$$
\begin{aligned}
& \Phi^{ \pm}=E^{ \pm}\left(E_{0}^{ \pm}\right)^{-1} F^{ \pm}, \quad E^{ \pm}=\left(\left(E_{0}^{ \pm}\right)^{-1} \pm \Pi\right)^{-1}, \\
& \Phi=F-F^{+} \Pi E^{+}\left(E_{0}^{+}\right)^{-1} F^{+},
\end{aligned}
$$

and

$$
\begin{aligned}
& \tilde{\Phi}^{ \pm}= \pm \tilde{E}^{ \pm} F^{ \pm}, \quad \tilde{E}^{ \pm}=\left(E_{0}^{ \pm} \pm \tilde{\Pi}\right)^{-1}, \\
& \tilde{\Phi}=F-F^{+} \tilde{E}^{+} F^{+},
\end{aligned}
$$

\footnotetext{
${ }^{2}$ In this paper explicit display of all the indices in the various symbols will be avoided whenever it causes no confusion.
} 
where $F_{i a}^{+}=F_{a i}^{-}, F_{a i}^{+}=F_{i a}^{-}$and $\left(E_{0}^{+}\right)_{a b}=\left(E_{0}^{-}\right)_{b a}$ may be at most functions of the variables $Y^{i}$ only. The common parametrization of $E_{A B}^{+}$and $\tilde{E}^{+A B}$ in terms of $E_{0}^{+}$ and $F_{a i}^{ \pm}$provides the required relation between them. Various aspects of Poisson-Lie T-duality can be found in [10, 11, 19, 20, 21, 11], generalization to $N=1$ world-sheet supersymmetric $\sigma$-models in [12, 22], and some relations to $N=2$ superconformal WZW models in [23].

\subsection{The proof}

In [12] it was conjectured, on the basis of symmetry arguments, and of a correct limit in the case of non-Abelian duality, when one of the groups $G$ or $\tilde{G}$ is Abelian, that the classical canonical transformation relating the $\sigma$-model actions (1) and (2) is given by

$$
\begin{aligned}
L_{\sigma} & =(I-\Pi \tilde{\Pi}) \tilde{P}-\Pi \tilde{L}_{\sigma}, \\
P & =\tilde{\Pi} \tilde{P}+\tilde{L}_{\sigma},
\end{aligned}
$$

with $P_{a}=L_{a}^{\mu} P_{\mu}$, where $P_{\mu}=\delta S / \delta \dot{X}^{\mu}$ is the conjugate to $X^{\mu}$ momentum, $L_{\sigma}^{a}=L_{\mu}^{a} \partial_{\sigma} X^{\mu}$ and similarly for the corresponding tilded symbols. We may cast (10) into the equivalent forms, which are in addition Poisson-Lie T-duality-symmetric,

$$
\begin{aligned}
& P=\tilde{\Pi} \tilde{P}+\tilde{L}_{\sigma}, \\
& \tilde{P}=\Pi P+L_{\sigma},
\end{aligned}
$$

and

$$
\begin{aligned}
P & =(I-\tilde{\Pi} \Pi)^{-1}\left(\tilde{L}_{\sigma}+\tilde{\Pi} L_{\sigma}\right), \\
\tilde{P} & =(I-\Pi \tilde{\Pi})^{-1}\left(L_{\sigma}+\Pi \tilde{L}_{\sigma}\right) .
\end{aligned}
$$

It was further checked [12 that (10) is good enough to prove that the Hamiltonians corresponding to actions (11) and (2) are equal, i.e. $H=\tilde{H}$. This was already a nontrivial check since the right-hand side of (10) depends on the $\tilde{X}^{\mu}$ 's and the $X^{\mu}$ 's and it is not a priori obvious that when (10) is used in transforming $H$ all $X^{\mu}$-dependence will cancel out, as it eventually does so. To complete the proof that one of (10)-(12) is indeed a canonical transformation, one has to derive it from a generating functional $F$ (with $\frac{\partial F}{\partial \tau}=0$ ) or equivalently prove that one of (10)-(12) leaves invariant the classical Poisson brackets of phase-space variables for (11) and (2). We found it more convenient to follow the latter approach, which we present now, leaving for later in the paper the discussion of the generating functional. First, let us focus on the action (1). The usual pair of phase-space variables $\left(X^{\mu}, P_{\nu}\right)$ satisfies the basic equal-time Poisson brackets]:

$$
\begin{aligned}
& \left\{X^{\mu}, P_{\nu}\right\}=\delta_{\nu}^{\mu} \delta\left(\sigma-\sigma^{\prime}\right), \\
& \left\{X^{\mu}, X^{\nu}\right\}=\left\{P_{\mu}, P_{\nu}\right\}=0 .
\end{aligned}
$$

\footnotetext{
${ }^{3}$ We will not explicitly display the world-sheet dependence of the phase-space variables involved in the various Poisson brackets. It is understood that the first one in the bracket is always evaluated at $\sigma$ and the second one at $\sigma^{\prime}$, whereas the $\tau$ dependence is common.
} 
Using them and the property of the Maurer-Cartan form $\partial_{[\mu} L_{\nu]}^{a}=f_{b c}{ }^{a} L_{\mu}^{b} L_{\nu}^{c}$, one computes the equal-time Poisson brackets for $\left(L_{\sigma}^{a}, P_{b}\right)$

$$
\begin{aligned}
\left\{L_{\sigma}^{a}, P_{b}\right\} & =f_{b c}{ }^{a} L_{\sigma}^{c} \delta\left(\sigma-\sigma^{\prime}\right)+\delta_{b}^{a} \delta^{\prime}\left(\sigma-\sigma^{\prime}\right) \\
\left\{P_{a}, P_{b}\right\} & =f_{a b}{ }^{c} P_{c} \delta\left(\sigma-\sigma^{\prime}\right) \\
\left\{L_{\sigma}^{a}, L_{\sigma^{\prime}}^{b}\right\} & =0 .
\end{aligned}
$$

A simple inspection of the equivalent transformations (10)-(12) shows that it will be convenient to consider Poisson brackets for $\left(J^{a}, P_{b}\right)$, where

$$
J^{a}=L_{\sigma}^{a}+\Pi^{a b} P_{b}
$$

The reason is that (11) then simply reads $P_{a}=\tilde{J}_{a}$ and $\tilde{P}^{a}=J^{a}$, with both sides of the transformation depending on variables defined in (1) or (2) only. Notice that this is not the case for (10) and (12). A relatively simple computation shows that

$$
\left\{J^{a}, P_{b}\right\}=\left(f_{b c}^{a} J^{c}+\left(f_{b d}{ }^{[a} \Pi^{c] d}+L_{b}^{\mu} \partial_{\mu} \Pi^{a c}\right) P_{c}\right) \delta\left(\sigma-\sigma^{\prime}\right)+\delta_{b}^{a} \delta^{\prime}\left(\sigma-\sigma^{\prime}\right),
$$

and

$$
\begin{aligned}
\left\{J^{a}, J^{b}\right\}= & -\left(f_{c d}{ }^{[a} \Pi^{b] d}+L_{c}^{\mu} \partial_{\mu} \Pi^{a b}\right) L_{\sigma}^{c} \delta\left(\sigma-\sigma^{\prime}\right) \\
& -\left(\Pi^{d b} \Pi^{e c} f_{d e}{ }^{a}+\tilde{f}^{a c}{ }_{d} \Pi^{b d}+\Pi^{a d} L_{d}^{\mu} \partial_{\mu} \Pi^{b c}\right) P_{c} \delta\left(\sigma-\sigma^{\prime}\right) .
\end{aligned}
$$

Using (A.6) in (16) and (A.7), (A.3) in (17) we find that they simplify to

$$
\begin{aligned}
& \left\{J^{a}, J^{b}\right\}=\tilde{f}^{a b}{ }_{c} J^{c} \delta\left(\sigma-\sigma^{\prime}\right), \\
& \left\{P_{a}, P_{b}\right\}=f_{a b}{ }^{c} P_{c} \delta\left(\sigma-\sigma^{\prime}\right), \\
& \left\{J^{a}, P_{b}\right\}=\left(f_{b c}{ }^{a} J^{c}-\tilde{f}_{b c}^{a c} P_{c}\right) \delta\left(\sigma-\sigma^{\prime}\right)+\delta_{b}{ }^{a} \delta^{\prime}\left(\sigma-\sigma^{\prime}\right),
\end{aligned}
$$

where we have carried over from (14) the expression for the Poisson bracket $\left\{P_{a}, P_{b}\right\}$. It is clear that the analogous equal-time Poisson brackets for the pair of phase-space variables $\left(\tilde{J}_{a}, \tilde{P}^{b}\right)$ defined for the action (2) are

$$
\begin{aligned}
\left\{\tilde{J}_{a}, \tilde{J}_{b}\right\} & =f_{a b}{ }^{c} \tilde{J}_{c} \delta\left(\sigma-\sigma^{\prime}\right) \\
\left\{\tilde{P}^{a}, \tilde{P}^{b}\right\} & =\tilde{f}^{a b}{ }_{c} \tilde{P}^{c} \delta\left(\sigma-\sigma^{\prime}\right) \\
\left\{\tilde{J}_{a}, \tilde{P}^{b}\right\} & =\left(\tilde{f}^{b c}{ }_{a} \tilde{J}_{c}-f_{a c}{ }^{b} \tilde{P}^{c}\right) \delta\left(\sigma-\sigma^{\prime}\right)+\delta_{a}{ }^{b} \delta^{\prime}\left(\sigma-\sigma^{\prime}\right) .
\end{aligned}
$$

Under the transformation $P_{a}=\tilde{J}_{a}, J^{a}=\tilde{P}^{a}$, one goes from (18) to (19) and vice versa, hence proving that (11) and equivalently (10) and (12) are indeed canonical transformations. We may view (18), (19) as the infinite-dimensional analogues of the group theory duality-invariant commutation relations (3), (4).

\footnotetext{
${ }^{4}$ Our symmetrization and antisymmetrization conventions are $(a b)=a b+b a$ and $[a b]=a b-b a$.
} 
In appendix B we find a Poisson-Lie T-duality invariant affine algebra using the manifestly Poisson-Lie T-duality Lorentz-non-invariant action of [11] as it was modified to consistently include the inert fields $Y^{i}$ in [12].

In subsection 3.2 we will extend the classical canonical equivalence to $N=1$ worldsheet supersymmetric $\sigma$-models related by Poisson-Lie T-duality by finding how the world-sheet fermions transform (see also [12]).

\section{$3 \quad$ On generating functionals}

As we have mentioned, it is not necessary to have a generating functional in order to prove that (10)-(12) are canonical transformations, since we have explicitly shown this to be the case at the Poisson bracket level. Nevertheless, it will be nice to obtain it, as it may provide more insight into Poisson-Lie T-duality as well as in searching for the quantum counterpart of the classical canonical transformation. We will be able to derive it perturbatively, in a sense to be explained shortly, but in the process we will provide the general setting for determining a generating functional for bosonic and supersymmetric models once a transformation between phase-space variables is given. For bosonic models there is some discussion in [24], but here we will be explicit.

\subsection{The bosonic case}

Let us consider two $\sigma$-models with actions

$$
S=\frac{1}{2} \int Q_{M N}^{+} \partial_{+} X^{M} \partial_{-} X^{N}, \quad Q_{M N}^{ \pm}=G_{M N} \pm B_{M N}, \quad X^{M}=\left(X^{\mu}, Y^{i}\right),
$$

and

$$
\tilde{S}=\frac{1}{2} \int \tilde{Q}_{M N}^{+} \partial_{+} \tilde{X}^{M} \partial_{-} \tilde{X}^{N}, \quad \tilde{Q}_{M N}^{ \pm}=\tilde{G}_{M N} \pm \tilde{B}_{M N}, \quad \tilde{X}^{M}=\left(\tilde{X}^{\mu}, Y^{i}\right),
$$

and a transformation that relates the conjugate momenta $P_{\mu}$ and $\tilde{P}_{\mu}$ to $X^{\mu}$ and $\tilde{X}^{\mu}$ as well as to their first derivatives $\partial_{\sigma} X^{\mu}$ and $\partial_{\sigma} \tilde{X}^{\mu}$. We do not include higher derivatives in $\sigma$ because such derivatives do not appear in (20), (21). The most general such transformation is of the form (see however remarks in section 4):

$$
\begin{aligned}
& P_{\mu}=A_{\mu \nu}(X, \tilde{X}) \partial_{\sigma} X^{\nu}+C_{\mu \nu}(X, \tilde{X}) \partial_{\sigma} \tilde{X}^{\nu} \\
& \tilde{P}_{\mu}=\tilde{A}_{\mu \nu}(X, \tilde{X}) \partial_{\sigma} \tilde{X}^{\nu}+\tilde{C}_{\mu \nu}(X, \tilde{X}) \partial_{\sigma} X^{\nu} .
\end{aligned}
$$

Then it is a canonical transformation if one can find a generating functional $F$ such that

$$
P_{\mu}=\frac{\delta F}{\delta X^{\mu}}, \quad \tilde{P}_{\mu}=-\frac{\delta F}{\delta \tilde{X}^{\mu}} .
$$


The generating functional should be of the form (we consider functionals with no explicit $\tau$-dependence):

$$
F=\oint d \sigma\left(B_{\mu}(X, \tilde{X}) \partial_{\sigma} X^{\mu}+\tilde{B}_{\mu}(X, \tilde{X}) \partial_{\sigma} \tilde{X}^{\mu}\right),
$$

for some functions $B_{\mu}, \tilde{B}_{\mu}$ of the target space variables that have to be determined. Using (23), (24) we find that (we will denote $\partial_{\mu}=\frac{\partial}{\partial X^{\mu}}$ and $\tilde{\partial}_{\mu}=\frac{\partial}{\partial \tilde{X}^{\mu}}$ )

$$
\begin{aligned}
& P_{\mu}=\partial_{[\mu} B_{\nu]} \partial_{\sigma} X^{\nu}+\left(\partial_{\mu} \tilde{B}_{\nu}-\tilde{\partial}_{\nu} B_{\mu}\right) \partial_{\sigma} \tilde{X}^{\nu}, \\
& \tilde{P}_{\mu}=-\tilde{\partial}_{[\mu} \tilde{B}_{\nu]} \partial_{\sigma} \tilde{X}^{\nu}-\left(\tilde{\partial}_{\mu} B_{\nu}-\partial_{\nu} \tilde{B}_{\mu}\right) \partial_{\sigma} X^{\nu} .
\end{aligned}
$$

Notice that $B_{\mu}$ and $\tilde{B}_{\mu}$ may be changed by total derivatives $\partial_{\mu} \Lambda_{2}$ and $\tilde{\partial}_{\mu} \Lambda_{2}$, respectively, with $\Lambda_{2}=\Lambda_{2}(X, \tilde{X})$, without affecting the generating functional (24) and the transformation (25). Next we compare (25) to (22) and obtain the following first-order differential equations for $B_{\mu}, \tilde{B}_{\mu}$

$$
\begin{aligned}
\partial_{[\mu} B_{\nu]} & =A_{\mu \nu}, \\
\tilde{\partial}_{[\mu} \tilde{B}_{\nu]} & =-\tilde{A}_{\mu \nu}, \\
\partial_{\mu} \tilde{B}_{\nu}-\tilde{\partial}_{\nu} B_{\mu} & =C_{\mu \nu}, \\
\tilde{\partial}_{\mu} B_{\nu}-\partial_{\nu} \tilde{B}_{\mu} & =-\tilde{C}_{\mu \nu} .
\end{aligned}
$$

One immediately sees? that the matrices in (22) should obey

$$
A_{\mu \nu}=-A_{\nu \mu}, \quad \tilde{A}_{\mu \nu}=-\tilde{A}_{\mu \nu}, \quad \tilde{C}_{\mu \nu}=C_{\nu \mu} .
$$

The latter condition makes (29) identical to (28). Further compatibility conditions for (26) -(29) may be easily derived by appropriately differentiating them and antisymmetrizing some indices

$$
\begin{aligned}
& \partial_{\mu} A_{\nu \rho}+\partial_{\rho} A_{\mu \nu}+\partial_{\nu} A_{\rho \mu}=0, \\
& \tilde{\partial}_{\mu} \tilde{A}_{\nu \rho}+\tilde{\partial}_{\rho} \tilde{A}_{\mu \nu}+\tilde{\partial}_{\nu} \tilde{A}_{\rho \mu}=0, \\
& \partial_{\mu} \tilde{A}_{\nu \rho}+\tilde{\partial}_{\nu} C_{\mu \rho}-\tilde{\partial}_{\rho} C_{\mu \nu}=0, \\
& \tilde{\partial}_{\mu} A_{\nu \rho}+\partial_{\nu} C_{\rho \mu}-\partial_{\rho} C_{\nu \mu}=0 .
\end{aligned}
$$

Equations (28), (29) considered as second-order equations for $B_{\mu}, \tilde{B}_{\mu}$ after using (26), (27), can be integrated once to give

$$
\tilde{\partial}_{\mu} B_{\nu}=-C_{\nu \mu}+\partial_{\nu} \tilde{\Gamma}_{\mu} .
$$

and

$$
\partial_{\mu} \tilde{B}_{\nu}=C_{\mu \nu}+\tilde{\partial}_{\nu} \Gamma_{\mu}
$$

\footnotetext{
${ }^{5}$ Note that, solving (26), (27) determines $B_{\mu}$ and $\tilde{B}_{\mu}$ up to total derivatives $\partial_{\mu} \Lambda_{1}(X, \tilde{X})$ and $\tilde{\partial}_{\mu} \Lambda(X, \tilde{X})$. We may consistently set $\Lambda_{1}=0$ since otherwise it may be absorbed into a redefinition of $\Lambda$, i.e. $\Lambda \rightarrow \Lambda+\Lambda_{1}$. The function $\Lambda$ is then determined by demanding that (28) is also satisfied.
} 
where $\tilde{\Gamma}_{\mu}, \Gamma_{\mu}$ are some unknown functions of $X^{\mu}, \tilde{X}^{\mu}$. Equations (35), (36) will be shortly used in deriving (43), (44) below, where the effect of $\tilde{\Gamma}_{\mu}, \Gamma_{\mu}$ will also be explained.

Finally, one considers the Hamiltonians corresponding to (20) and (21). Namely,

$$
H=\frac{1}{2} \oint d \sigma\left(G^{M N}\left(P_{M}+B_{M \Lambda} \partial_{\sigma} X^{\Lambda}\right)\left(P_{N}+B_{N P} \partial_{\sigma} X^{P}\right)+G_{M N} \partial_{\sigma} X^{M} \partial_{\sigma} X^{N}\right)
$$

and a similar expression for $\tilde{H}$. For (22) to be a canonical transformation, one must require that apart from the fact that it can be derived from a generating functional using (23), the Hamiltonians for (20), (21) must be equal, i.e. $H=\tilde{H}$. After some tensor manipulations we find that this is guaranteed, provided that the following conditions hold

$$
\begin{aligned}
& \left(C^{-1}\right)^{\mu \rho}\left(Q_{\rho \lambda}^{ \pm} \pm A_{\rho \lambda}\right)\left(C^{-1}\right)^{\kappa \lambda}\left(\tilde{Q}_{\kappa \nu}^{ \pm} \pm \tilde{A}_{\kappa \nu}\right)=\delta_{\nu}^{\mu}, \\
& Q_{\mu i}^{ \pm}=\left(Q_{\mu \rho}^{ \pm} \pm A_{\mu \rho}\right)\left(C^{-1}\right)^{\lambda \rho} \tilde{Q}_{\lambda i}^{ \pm} .
\end{aligned}
$$

Hence, knowledge of the canonical transformation and of $Q_{M N}^{ \pm}\left(\tilde{Q}_{M N}^{ \pm}\right)$determines $\tilde{Q}_{M N}^{ \pm}$ $\left(Q_{M N}^{ \pm}\right)$.

Let us return to the case of Poisson-Lie T-duality. Then, as it was mentioned, the $X^{\mu}$ 's and the $\tilde{X}^{\mu}$ 's parametrize the groups $G$ and $\tilde{G}$ respectively. It is therefore natural to use, instead of $A_{\mu \nu}, \tilde{A}_{\mu \nu}$ and $C_{\mu \nu}$, matrices with group-space indices defined as

$$
A_{\mu \nu}=A_{a b} L_{\mu}^{a} L_{\nu}^{b}, \quad \tilde{A}_{\mu \nu}=\tilde{A}^{a b} \tilde{L}_{\mu a} \tilde{L}_{\nu b}, \quad C_{\mu \nu}=C_{a}^{b} L_{\mu}^{a} \tilde{L}_{\nu b} ;
$$

also, we identify $E_{A B}^{ \pm}=L_{M}^{A} L_{N}^{B} Q_{M N}^{ \pm}$and $\tilde{E}^{ \pm A B}=\tilde{L}^{M A} \tilde{L}^{N B} \tilde{Q}_{M N}^{ \pm}$. Then comparing (12) and (22) we read off the matrices $A_{a b}, \tilde{A}^{a b}, C_{a}{ }^{b}$ and $\tilde{C}^{a}{ }_{b}$ as

$$
\begin{array}{lll}
A=(I-\tilde{\Pi} \Pi)^{-1} \tilde{\Pi}, & \tilde{A}=(I-\Pi \tilde{\Pi})^{-1} \Pi, \\
C=(I-\tilde{\Pi} \Pi)^{-1}, & \tilde{C}=(I-\Pi \tilde{\Pi})^{-1} .
\end{array}
$$

It can be easily seen that (30) and (38) are indeed satisfied. Solving the differential equations (26)-(28) turned out to be a much more difficult task. We found it convenient to use the parametrization for the group element $\tilde{g}=e^{i \tilde{X}_{a} \tilde{T}^{a}}$ and hence the target-space variables for the action (2) are the $\tilde{X}_{a}$ 's. Then it turns out that

$$
\begin{aligned}
& \tilde{a}=e^{-\tilde{f}}=I-\tilde{f}+\frac{1}{2} \tilde{f}^{2}+\ldots \\
& \tilde{b}=\sum_{n=0}^{\infty} \sum_{m=0}^{n} \frac{(-1)^{m}}{n !}\left(\tilde{f}^{T}\right)^{n-m} f \tilde{f}^{m}=f-\frac{1}{2}\left(f \tilde{f}-\tilde{f}^{T} f\right)+\ldots \\
& \tilde{\Pi}=\sum_{n=0}^{\infty} \sum_{m=0}^{n} \frac{(-1)^{n+1}}{(n+1) m !(n-m) !}\left(\tilde{f}^{T}\right)^{n-m} f \tilde{f}^{m}=-f+\frac{1}{2}\left(f \tilde{f}+\tilde{f}^{T} f\right)+\ldots \\
& \tilde{L}=\left(I-e^{-\tilde{f}}\right) / \tilde{f}=I-\frac{1}{2} \tilde{f}+\frac{1}{6} \tilde{f}^{2}+\ldots,
\end{aligned}
$$

\footnotetext{
${ }^{6}$ For a general element $l$ of a group $D$ (with algebra generators $T_{A}$ ) parametrized as $l=e^{i T_{A} X^{A}}$, one has [25] $l^{-1} T_{A} l=\left(e^{-F}\right)_{A}{ }^{B} T_{B}$ and $L=\left(1-e^{-F}\right) / F$, where $F_{A}{ }^{B}=F_{A C}{ }^{B} X^{C}$. In our case the group $D$ is the Drinfeld double, $l=\tilde{g}$ and $T_{A}=\left\{T_{a}, \tilde{T}^{a}\right\}$. Then using (6) one reads off the matrix $F=\left(\begin{array}{cc}-\tilde{f}^{T} & -f \\ 0 & \tilde{f}\end{array}\right)$, from which one computes $e^{-F}$ and hence $\tilde{a}, \tilde{b}, \tilde{\Pi}$ and $\tilde{L}$.
} 
where the matrices $f$ and $\tilde{f}$ have components

$$
f_{a b}=f_{a b}{ }^{c} \tilde{X}_{c}, \quad \tilde{f}_{b}^{a}=\tilde{f}^{a c}{ }_{b} \tilde{X}_{c} .
$$

Similar expressions hold for the untilded quantities in the parametrization of $g=e^{i X^{a} T_{a}}$. Equations (26) and (27) can be solved as a power series in the $\tilde{X}_{a}$ 's. In fact we may find a closed formula for $B_{\mu}$ by using (35) with (40) to evaluate the higher-order derivatives in the Taylor expansion of $B_{\mu}$ in powers of the $\tilde{X}_{a}$ 's. The result is

$$
B_{\mu}=-L_{\mu}^{a} \int_{0}^{1} d t C_{a}^{b}(X, t \tilde{X}) \tilde{X}_{b}
$$

where in the matrix $C$, defined in (40), all the $\tilde{X}_{a}$ 's contained in the expression for $\tilde{\Pi}$ in (41) are replaced by $t \tilde{X}_{a}$ 's. Notice also that in accordance with the remaks in footnote 5 we have dropped the contribution of the function $\tilde{\Gamma}_{\mu}$, since it is a $\partial_{\mu}$-derivative. A result similar to (43) holds for $\tilde{B}_{\mu}$

$$
\tilde{B}_{\mu}=\tilde{L}_{\mu a} \int_{0}^{1} d t C_{b}{ }^{a}(t X, \tilde{X}) X^{b}+\tilde{\partial}_{\mu} \Lambda(X, \tilde{X}),
$$

where we have now replaced in $C$ all the $X^{a}$ 's contained in the expression for $\Pi$ by $t X^{a}$ 's.' The function $\Lambda(X, \tilde{X})$ is related to $\Gamma_{\mu}$ in (36) and is to be determined perturbatively by demanding that (28) is also satisfied. It seems helpless to exactly perform the integral in (43) (and in (44)) because the $t$-dependence of $C(X, t \tilde{X})$ is quite complicated. To order $O(\tilde{X})^{2}$, but exactly in the $X^{\mu}$ variables, we find

$$
\begin{aligned}
& B_{\mu}=L_{\mu}^{a}\left(-\tilde{X}_{a}+\frac{1}{2} f_{a b} \Pi^{b c} \tilde{X}_{c}+\ldots\right), \\
& \tilde{B}_{\mu}=\frac{1}{2} \Pi^{\mu a} \tilde{X}_{a}+\frac{1}{6} \Lambda^{a b \mu} \tilde{X}_{a} \tilde{X}_{b}+\ldots,
\end{aligned}
$$

where $\Lambda^{a b \mu}$ is defined in (A.9). In the case of non-Abelian duality where the group $\tilde{G}$ (equivalently $G$ ) is Abelian, (46) is reduced to $B_{\mu}=-L_{\mu}^{a} \tilde{X}_{a}, \tilde{B}_{a}=0$ (since then $\left.\tilde{f}_{c}^{a b}=\Pi^{a b}=0\right)$, which in fact gives the exact results for the generating functional in that case [6, 8, 9]. Hence, the extra terms in (46) represent in some sense the deviations of Poisson-Lie T-duality from the usual non-Abelian duality. When the group $G$ is also Abelian, (46) is further reduced to $B_{a}=-\tilde{X}_{a}, \tilde{B}_{a}=0$, which of course corresponds to the exact generating functional for Abelian duality [7]. Let us also note that since the generating functional in highly non-linear in $X^{\mu}, \tilde{X}^{\mu}$, it will presumably receive quantum corrections when the canonical transformation is implemented in the full Hilbert space along the lines of [26].

\footnotetext{
${ }^{7}$ We may easily write (43), (44) in an arbitrary coordinate system by just expressing $\tilde{X}_{a}$ and $X^{a}$ in terms of the corresponding group elements $\tilde{g}$ and $g$ respectively. For instance, (43) takes the form

$$
B_{\mu}=i L_{\mu}^{a} \int_{0}^{1} d t C_{a}^{b}\left(g, \tilde{g}^{t}\right)\left\langle\ln \tilde{g} \mid T_{b}\right\rangle
$$

where instead of the group element $\tilde{g}$ in the definition of $C$ in (40) we must use its power $\tilde{g}^{t}$. A similar expression holds for (44) as well.
} 


\subsection{The supersymmetric case}

We would like to extend the previous discussion to $N=1$ supersymmetric $\sigma$-models, with actions corresponding to (20), (21) given by

$$
\begin{aligned}
& S_{N=1}=\frac{1}{2} \int Q_{M N}^{+} \partial_{+} X^{M} \partial_{-} X^{N}+i Q_{M N}^{+} \Psi_{+}^{M} \partial_{-} \Psi_{+}^{N}+i Q_{M N}^{-} \Psi_{-}^{M} \partial_{+} \Psi_{-}^{N} \\
& \quad+i S_{M ; \Lambda N}^{+} \Psi_{+}^{M} \Psi_{+}^{N} \partial_{-} X^{\Lambda}+i S_{M ; \Lambda N}^{-} \Psi_{-}^{M} \Psi_{-}^{N} \partial_{+} X^{\Lambda}+\frac{1}{2} R_{M N P \Lambda}^{-} \Psi_{+}^{M} \Psi_{+}^{N} \Psi_{-}^{P} \Psi_{-}^{\Lambda}
\end{aligned}
$$

where $S_{M ; \Lambda N}^{ \pm}=\frac{1}{2} \partial_{[N} Q_{M] \Lambda}^{ \pm}$and $R_{M N P \Lambda}^{-}$is the generalized curvature tensor. $]$A similar expression holds for $\tilde{S}_{N=1}$ as well. The generating functional is easily obtained by supersymmetrizing the bosonic one (24). This is done by simply replacing the bosonic fields and derivatives by their supersymmetric counterparts. However, in order to do that we first have to take the $\tau$-derivative of (24) because this is how it relates the Lagrangians by definition, i.e. $\mathcal{L}=\tilde{\mathcal{L}}+\frac{d F}{d \tau}$. Then one obtains

$$
\begin{gathered}
\frac{d F_{N=1}}{d \tau}=-\frac{1}{2} \oint d \\
+C_{\mu \nu}\left(D_{+} Z^{\mu} D_{-} d \theta_{+}\left(A_{\mu \nu} D_{+} Z^{\mu} D_{-} Z^{\nu}-D_{+} \tilde{Z}_{\mu \nu} D_{+} D_{-} Z^{\mu}\right)\right) \\
\tilde{Z}^{\mu} D_{-} \tilde{Z}^{\nu} \\
\end{gathered}
$$

where we have used (26)-(28). The (bosonic) superfield and superderivatives are given by

$$
\begin{aligned}
& Z^{M}=X^{M}-i \theta_{+} \Psi_{-}^{M}+i \theta_{-} \Psi_{+}^{M}-i \theta_{+} \theta_{-} F^{M}, \\
& D_{ \pm}=\mp i \partial_{\theta_{\mp}} \mp \theta_{\mp} \partial_{ \pm},
\end{aligned}
$$

with $F^{M}=i \Omega_{N \Lambda}^{+M} \Psi_{-}^{N} \Psi_{+}^{\Lambda}$ on shell, where $\Omega_{N \Lambda}^{+M}$ is the generalized connection that includes the torsion $H_{M N \Lambda}=\partial_{[M} B_{N \Lambda]}$ in its definition. An expression similar to (50) holds for $\tilde{Z}^{M}$ as well. After we perform the $\theta_{ \pm}$integration we are left with an integrand that can be written, after some tensor manipulations, as a total derivative in $\tau$. From that we extract the generating functional, which assumes the form

$$
\begin{aligned}
F_{N=1}=\oint d \sigma & \left(B_{\mu} \partial_{\sigma} X^{\mu}+\tilde{B}_{\mu} \partial_{\sigma} \tilde{X}^{\mu}-\frac{i}{4} A_{\mu \nu}\left(\Psi_{+}^{\mu} \Psi_{+}^{\nu}-\Psi_{-}^{\mu} \Psi_{-}^{\nu}\right)\right. \\
& \left.+\frac{i}{4} \tilde{A}_{\mu \nu}\left(\tilde{\Psi}_{+}^{\mu} \tilde{\Psi}_{+}^{\nu}-\tilde{\Psi}_{-}^{\mu} \tilde{\Psi}_{-}^{\nu}\right)-\frac{i}{2} C_{\mu \nu}\left(\Psi_{+}^{\mu} \tilde{\Psi}_{+}^{\nu}-\Psi_{-}^{\mu} \tilde{\Psi}_{-}^{\nu}\right)\right) .
\end{aligned}
$$

\footnotetext{
${ }^{8}$ Action (47) appears slightly different from the standard expressions found in the literature (see for instance [27]). The reason is that we have retained the total derivative terms$$
\Delta S=-\frac{i}{2} \int \partial_{+}\left(B_{M N} \Psi_{-}^{M} \Psi_{-}^{N}\right)-\partial_{-}\left(B_{M N} \Psi_{+}^{M} \Psi_{+}^{N}\right)
$$

which are usually dropped in passing from the superfield to the component formalism of $N=1$ supersymmetry. We may verify that if we subtract (48) from (47) we get the usual form of the action. This point was appreciated in the second of refs. 28.
} 
Notice that the fermionic terms in the above expression are completely determined by the matrices entering into the bosonic transformations (22). Using (51), (23) the transformation rules for $P_{\mu}, \tilde{P}_{\mu}$ are found to be

$$
\begin{aligned}
P_{\mu}= & A_{\mu \nu} \partial_{\sigma} X^{\nu}+C_{\mu \nu} \partial_{\sigma} \tilde{X}^{\nu}-\frac{i}{4} \partial_{\mu} A_{\nu \rho}\left(\Psi_{+}^{\nu} \Psi_{+}^{\rho}-\Psi_{-}^{\nu} \Psi_{-}^{\rho}\right) \\
& +\frac{i}{4} \partial_{\mu} \tilde{A}_{\nu \rho}\left(\tilde{\Psi}_{+}^{\nu} \tilde{\Psi}_{+}^{\rho}-\tilde{\Psi}_{-}^{\nu} \tilde{\Psi}_{-}^{\rho}\right)-\frac{i}{2} \partial_{\mu} C_{\nu \rho}\left(\Psi_{+}^{\nu} \tilde{\Psi}_{+}^{\rho}-\Psi_{-}^{\nu} \tilde{\Psi}_{-}^{\rho}\right),
\end{aligned}
$$

with a similar expression for $\tilde{P}_{\mu}$. The transformation rules for fermions are found by using

$$
P_{\Psi_{ \pm}^{\mu}}=\frac{\delta F_{N=1}}{\delta \Psi_{ \pm}^{\mu}}, \quad P_{\tilde{\Psi}_{ \pm}^{\mu}}=\frac{\delta F_{N=1}}{\delta \tilde{\Psi}_{ \pm}^{\mu}},
$$

and the explicit expressions for the conjugate to the fermions momenta, which are given by

$$
\begin{aligned}
& P_{\Psi_{ \pm}^{\mu}}=\frac{\delta S_{N=1}}{\delta \Psi_{ \pm}^{\mu}}=-\frac{i}{2}\left(Q_{\mu \nu}^{\mp} \Psi_{ \pm}^{\nu}+Q_{\mu i}^{\mp} \Psi_{ \pm}^{i}\right) \\
& P_{\tilde{\Psi}_{ \pm}^{\mu}}=\frac{\delta S_{N=1}}{\delta \tilde{\Psi}_{ \pm}^{\mu}}=-\frac{i}{2}\left(\tilde{Q}_{\mu \nu}^{\mp} \tilde{\Psi}_{ \pm}^{\nu}+Q_{\mu i}^{\mp} \Psi_{ \pm}^{i}\right) .
\end{aligned}
$$

The result is

$$
\begin{aligned}
& \tilde{\Psi}_{ \pm}^{\mu}= \pm\left(C^{-1}\right)^{\mu \nu}\left(\left(Q_{\nu \rho}^{\mp} \mp A_{\nu \rho}\right) \Psi_{ \pm}^{\rho}+Q_{\nu i}^{\mp} \Psi_{ \pm}^{i}\right) \\
& \Psi_{ \pm}^{\mu}= \pm\left(C^{-1}\right)^{\nu \mu}\left(\left(\tilde{Q}_{\nu \rho}^{\mp} \mp \tilde{A}_{\nu \rho}\right) \tilde{\Psi}_{ \pm}^{\rho}+\tilde{Q}_{\nu i}^{\mp} \Psi_{ \pm}^{i}\right) .
\end{aligned}
$$

Consistency of the two transformations is guaranteed by (38). In the case of Poisson-Lie

T-duality, it is natural to use fermions with tangent-space indices, i.e. $\Psi_{ \pm}^{a}=\Psi_{ \pm}^{\mu} L_{\mu}^{a}$ and $\tilde{\Psi}_{ \pm a}=\tilde{\Psi}_{ \pm}^{\mu} \tilde{L}_{\mu a}$. Then using (39) we may easily show that (55) reduces to eq. (40) of [12]. In the case of non-Abelian duality using the fact that $\Pi^{a b}=0, \tilde{\Pi}_{a b}=-f_{a b}{ }^{c} \tilde{X}_{c}$, the generating functional (52) reduces to

$$
F_{N=1}^{n . a .}=\oint d \sigma\left(L_{\sigma}^{a} \tilde{X}_{a}+\frac{i}{4} f_{a b}{ }^{c} \tilde{X}_{c}\left(\Psi_{+}^{a} \Psi_{+}^{b}-\Psi_{-}^{a} \Psi_{-}^{b}\right)-\frac{i}{2}\left(\Psi_{+}^{a} \tilde{\Psi}_{+a}-\Psi_{-}^{a} \tilde{\Psi}_{-a}\right)\right)
$$

which has been used in [28].

\section{Concluding remarks and future directions}

We explicitly formulated Poisson-Lie T-duality as a canonical transformation on the world-sheet by proving that a certain transformation, conjectured in [12], between phasespace variables does indeed leave the Poisson brackets invariant. Since the corresponding $\sigma$-models do not generically have any isometries, we have provided the first example of a 
class of models that are non-isometric but still classically canonically equivalent.? Furthermore we have presented a general discussion on generating functionals of canonical transformations in bosonic and $N=1$ supersymmetric $\sigma$-models and derived the complete set of conditions that determine them. Applying the general formalism to the case of Poisson-Lie T-duality, we derived perturbatively, in a sense that was explained, the corresponding generating functional.

Although not immediately obvious, the form of the canonical transformation (22) is not the most general one. The reason is that we have excluded Wilson lines from appearing in the right-hand sides. A necessary condition for Wilson lines to be permissible is that they cancel each other when the transformation is implemented into the Hamiltonian, which is bilinear. It is clear that in its usual formulation the generating functional approach to canonical transformations (cf. (23), (24)) cannot accommodate these more general transformations, but the canonical equivalence can still be shown by examining the invariance of the Poisson brackets. An important example of such a canonical transformation was given in [9] for the WZW model for a group $G$ and its non-Abelian dual with respect to the vector action of a subgroup $H$. In this case the canonical mapping for the group element $g \in G$ is

$$
g \rightarrow h_{-}^{-1} g h_{-}, \quad h_{-}=P e^{-\int^{\sigma^{-}} A_{-}},
$$

where $P$ stands for path ordering, and $A_{-}$is identified with the on-shell value of the gauge field in the standard approach with Langrange multipliers and it depends on $g$ as well as on the Lagrange multipliers themselves. In the right-hand side of (57) (including $A_{-}$) we keep only those parameters in $g \in G$ and among the Lagrange multipliers that are gaugeinvariant (this is equivalent to gauge fixing $\operatorname{dim}(H)$ out of the total $\operatorname{dim}(G)+\operatorname{dim}(H)$ parameters). Then it turns out [9] that the local chiral and anti-chiral currents of the WZW model become parafermionic-like objects of the same chirality, with Wilson lines attached to them. Since the conjugate momenta in the WZW model are related linearly to the chiral and anti-chiral currents, it is clear that Wilson lines will appear in the righthand side of (22). Nevertheless, the Hamiltonian is still local in the dual target-space variables and the affine current Poisson bracket algebra of the original WZW model is indeed respected [9]. It seems plausible that a canonical formulation of the so-called quasi axial-vector duality [30] and of the non-Abelian duality in PCMs with respect to the vectorial action of the group, will utilize these more general transformations. A detailed account of these and related issues is interesting but beyond the scope of the present paper.

Our results are useful in discussing T-duality for open strings in D-manifolds. Firstly, by knowing the explicit canonical map in phase space for Poisson-Lie T-duality, we may study the mapping of the boundary conditions in a systematic way. This will generalize and unify previous work on Abelian and non-Abelian duality for open strings

\footnotetext{
${ }^{9}$ This is similar in spirit to being able to perform a duality transformation in antisymmetric tensor theories in the presence of magnetic sources or monopole condensates, where also global symmetries are broken (see for instance 29]). However, a canonical formulation in these cases is lacking.
} 
16, 15, 31 and also complement and clarify existing work [21] on D-branes and PoissonLie T-duality. Perhaps more importantly, we may study T-duality when the backreaction string loop effects into the backgrounds fields due to the D-branes are taking into account. These are due to a Fischler-Susskind type mechanism [17] which in general doesn't respect the isometries the original backgrounds might have, hence rending the discussion within the usual context of Abelian and non-Abelian T-duality as inappropriate. Whether or not the backreaction-corrected backgrounds will be related, at least in some cases, by Poisson-Lie T-duality is an open problem;there is a priori no reason why they will be so. However, if this is the case, the corrections to the generating functional we are after (for the case of Abelian or non-Abelian duality for open strings) should take the form of the correction terms in (46). Even if the backreaction-corrected backgrounds are not Poisson-Lie T-duality-related, we still believe that our quite general (proviso the remarks of the previous paragraph) treatment of generating functionals will be useful in studying their possible canonical equivalence and hence shed more light into D-brane physics. However, there could be an interesting twist in this: since T-duality is valid at each order in the string coupling perturbation theory and since the backreaction effects arise from a higher order in the corresponding expansion than the original background, it is not clear whether or not T-duality is preserved by the combined effect or it is replaced by some other symmetry. Seeds of such symmetry (if it exists at all) should be found in a non-perturbative formulation of string theory. It is very much worth investigating these issues further.

For generic backgrounds the T-duality rules have to be corrected order by order in the $\sigma$-model coupling constant perturbation theory. This was first shown for the case of Abelian duality in a restricted class of conformal backgrounds [32. In 33] a careful analysis on the equivalence of $\sigma$-models, related classically by Abelian and non-Abelian T-duality and treated as 2-dimensional field theories in their own right, was carried out. The primary example used in 33] was a 1-parameter family model interpolating between the $S U(2) \mathrm{PCM}$ and the $O(3) \sigma$-model (2-sphere). The upshot of their analysis was that for Abelian duality the equivalence holds at 1 loop, but at the 2-loop level it requires that the classical transformation be corrected, in accordance in spirit with 32. For non-Abelian duality, a similar statement holds as well. One should perform a similar analysis in an example where Poisson-Lie T-duality is non-trivially realized, i.e. it does not reduce to the usual Abelian or non-Abelian duality. The analysis of [33] did not include counterterms that would have spoiled the isometries that the classical action had. It will be interesting to investigate this issue in the spirit of Poisson-Lie T-duality, or even the more general ones discussed in section 3, by allowing certain counterterms that will be admissible in a more general framework, even though they will break isometries. Perhaps Abelian and non-Abelian duality on generic curved backgrounds are meaningful only classically and quantum effects force us to work with more general models, with less isometries. A related issue is the preservation or not of conformal invariance in string backgrounds under Poisson-Lie T-duality, which is related to the transformation properties of the dilaton. 
Our final brief remark concerns mirror symmetry and T-duality in Calabi-Yau compactifications. Since Calabi-Yau manifolds have no isometries, in order to prove mirror symmetry as T-duality, it seems imperative to understand T-duality in the absence of isometries. Our results on Poisson-Lie T-duality and especially our general formulation of T-duality in non-isometric backgrounds might be helpful towards proving this conjecture.

\section{A Derivation of useful identities}

In this appendix we prove some useful identities by using mainly (6) and (7). All of them have their corresponding "duals", obtained by just replacing tilded symbols by untilded ones and vice versa. We start by explicitly evaluating $\left\langle g^{-1} T_{b} g \mid\left[\tilde{T}^{a}, g^{-1} T_{c} g\right]\right\rangle=$ $\left\langle\tilde{T}^{a} \mid g^{-1}\left[T_{c}, T_{b}\right] g\right\rangle$. We find the identity

$$
a_{b}^{c} a_{c}{ }^{d} f_{c d}^{a}=f_{b c}{ }^{d} a_{d}^{a} .
$$

Next we evaluate $\left\langle g T_{b} g^{-1} \mid\left[\tilde{T}^{a}, g \tilde{T}^{c} g^{-1}\right]\right\rangle=\left\langle\tilde{T}^{a} \mid g\left[\tilde{T}^{c}, T_{b}\right] g^{-1}\right\rangle$ and contract the result with $\left(a^{-1}\right)_{a}{ }^{d}$ to find, after we use (A.1) and relabel the indices, the identity

$$
f_{c d}\left[{ }^{a} \Pi^{b] d}+\tilde{f}^{a b}{ }_{c}=\tilde{f}_{f}^{d e}\left(a^{-1}\right)_{c}^{f} a_{d}^{a} a_{e}^{b} .\right.
$$

Another identity is derived by evaluating $\left\langle g^{-1} \tilde{T}^{b} g \mid\left[\tilde{T}^{a}, g^{-1} \tilde{T}^{c} g\right]\right\rangle=\left\langle\tilde{T}^{a} \mid g^{-1}\left[\tilde{T}^{c}, \tilde{T}^{b}\right] g\right\rangle$ and contracting the result by $a_{b}{ }^{d} a_{c}{ }^{e}$. We find that

$$
f_{d e}^{a} \Pi^{d b} \Pi^{e c}-\tilde{f}^{a[b}{ }_{d} \Pi^{c] d}=\tilde{f}^{d e}{ }_{f} b^{f a} a_{d}{ }^{b} a_{e}{ }^{c} .
$$

Using the definitions (6) we compute the derivatives

$$
\begin{aligned}
& \partial_{\mu} a_{a}^{b}=-a_{a}{ }^{c} L_{\mu}^{d} f_{c d}{ }^{b}, \\
& \partial_{\mu} b^{a b}=-L_{\mu}^{d}\left(b^{a c} f_{c d}{ }^{b}+\left(a^{-1}\right)_{c}{ }^{a} \tilde{f}_{d}^{b c}\right),
\end{aligned}
$$

and then

$$
\partial_{\mu} \Pi^{a b}=-L_{\mu}^{c}\left(\tilde{f}_{c}^{a b}+f_{c d}\left[a \Pi^{b] d}\right) .\right.
$$

For the latter there is an alternative form found by using (A.2). It reads

$$
\partial_{\mu} \Pi^{a b}=-L_{\mu}^{c} \tilde{f}_{f}^{d e}\left(a^{-1}\right)_{c}^{f} a_{d}^{a} a_{e}^{b} .
$$

Another useful identity is derived by contracting (A.2) by $\Pi^{d c}$ and then antisymmetrizing in $b, d$ and using the definition (6) and the identity (A.3). The result is the cyclic identity

$$
\Lambda^{[a b c]} \equiv \Lambda^{a b c}+\Lambda^{c a b}+\Lambda^{b c a}=0
$$

where

$$
\Lambda^{a b c}=\tilde{f}^{a[b}{ }_{d} \Pi^{c] d}-2 f_{d e}^{a} \Pi^{d b} \Pi^{e c} .
$$

It turns out that (A.8) is crucial in establishing the second of eqs. (46). Let us also mention that more general identities can be found by replacing the group element $g \in G$ at the starting point, by a general group element $l \in D$. 


\section{B Manifestly duality-invariant affine algebra}

In this appendix we consider the manifestly Poisson-Lie T-duality-invariant action of [11, with the inclusion of the extra inert fields $Y^{i}$ [12], and derive a Poisson bracket affine algebra, which is the analogue of (18), (19). We will use the notation and definitions of [12], which we will not repeat here. The action in question in given by eq. (26) of [12], which we rewrite in the form

$$
\begin{aligned}
S=I_{0}(l) & +\frac{1}{2 \pi} \int\left\langle l^{-1} \partial_{\sigma} l|R| l^{-1} \partial_{\sigma} l\right\rangle-2 i\left\langle l^{-1} \partial_{\sigma} l \mid F_{i}\right\rangle \partial_{\tau} Y^{i}+2 i\left\langle l^{-1} \partial_{\sigma} l|R| F_{i}\right\rangle \partial_{\sigma} Y^{i} \\
& +\left\langle F_{i} \mid F_{j}\right\rangle \partial_{\sigma} Y^{i} \partial_{\tau} Y^{j}-\left\langle F_{i}|R| F_{j}\right\rangle \partial_{\sigma} Y^{i} \partial_{\sigma} Y^{j} \\
& +\left(F_{i j}+\left\langle F_{i} \mid R_{a}^{-}\right\rangle \eta^{a b}\left\langle R_{b}^{+} \mid F_{j}\right\rangle-\frac{1}{2}\left\langle F_{i}|R| F_{j}\right\rangle\right) \partial_{+} Y^{i} \partial_{-} Y^{j}
\end{aligned}
$$

where we have defined

$$
F_{i}=F_{a i}^{+} \eta^{a b} R_{b}^{+}+F_{a i}^{-} \eta^{a b} R_{b}^{-} .
$$

The relation of (B.1) to (11), (2) is a follows [11, 12]: In the vicinity of the unit element of $D$ we may decompose [18 the group element $l \in D$ as $l=\tilde{h} g$ or as $l=h \tilde{g}$, where $h, g \in G$ and $\tilde{h}, \tilde{g} \in \tilde{G}$. Using the first decomposition we may integrate out $\tilde{h}$ using the equations of motion to solve for the corresponding "gauge" field $A_{ \pm}=\tilde{h}^{-1} \partial_{ \pm} \tilde{h} \in \tilde{\mathcal{G}}$. The result is action (11). Using the second decomposition and integrating out $h$ by solving for $\tilde{A}_{ \pm}=h^{-1} \partial_{ \pm} h \in \mathcal{G}$ we obtain instead action (2). Action (B.1) is first order in $\tau$ as far as $l$ are concerned. Hence, in order to find the corresponding Poisson brackets we have to apply Dirac's procedure. This is quite straightforward with the result

$$
\begin{aligned}
& \left\{\left\langle\delta l l^{-1} \mid R_{a}^{ \pm}\right\rangle,\left\langle\delta l l^{-1} \mid R_{b}^{ \pm}\right\rangle\right\}=\mp \frac{\pi}{2} \eta_{a b} \epsilon\left(\sigma-\sigma^{\prime}\right), \\
& \left\{\left\langle\delta l l^{-1} \mid R_{a}^{+}\right\rangle,\left\langle\delta l l^{-1} \mid R_{b}^{-}\right\rangle\right\}=0
\end{aligned}
$$

where $\epsilon\left(\sigma-\sigma^{\prime}\right)$ is a step function defined as $+1(-1)$ if $\sigma-\sigma^{\prime}>0\left(\sigma-\sigma^{\prime}<0\right)$. Then if we define the current

$$
J=i l^{-1} \partial_{\sigma} l=J_{a}^{+} \eta^{a b} R_{b}^{+}-J_{a}^{-} \eta^{a b} R_{b}^{-},
$$

we may obtain the Poisson brackets for the components $J_{a}^{ \pm}$as

$$
\begin{aligned}
& \left\{J_{a}^{ \pm}, J_{b}^{ \pm}\right\}=-i \pi\left\langle\left[R_{a}^{ \pm}, R_{b}^{ \pm}\right] \mid J\right\rangle \delta\left(\sigma-\sigma^{\prime}\right) \mp \pi \eta_{a b} \delta^{\prime}\left(\sigma-\sigma^{\prime}\right), \\
& \left\{J_{a}^{+}, J_{b}^{-}\right\}=-i \pi\left\langle\left[R_{a}^{+}, R_{b}^{-}\right] \mid J\right\rangle \delta\left(\sigma-\sigma^{\prime}\right) .
\end{aligned}
$$

The above algebra is manifestly Poisson-Lie T-duality-invariant. We note that (see also [12]) the canonical transformation (10) and the requirement for 2-dimensional Lorentz invariance on the world-sheet implies (8) and (9) as well as the equality

$$
\left.J_{a}^{ \pm}\right|_{A_{ \pm}} ^{l=\tilde{h} g}=\left.J_{a}^{ \pm}\right|_{\tilde{A}_{ \pm}} ^{l=h \tilde{g}}
$$

where in the left-hand side we parametrized $l \in D$ as denoted and inserted the classical value of $A_{ \pm}$, and similarly in the right-hand side. Vice versa, one can show that (B.6) implies the canonical transformation (10). 


\section{References}

[1] K. Kikkawa and M. Yamasaki, Phys. Lett. B149 (1984) 357; N. Sakai and I. Senda, Prog. Theor. Phys. 75 (1986) 692; T. Buscher, Phys. Lett. B194 (1987) 59 and B201 (1988) 466.

[2] A. Giveon, E. Rabinovici and G. Veneziano, Nucl. Phys. B322 (1989) 167; K. Meissner and G. Veneziano, Phys. Lett. B267 (1991) 33.

[3] M. Rocek and E. Verlinde, Nucl. Phys. B373 (1992) 630 hep-th/9110053; A. Giveon and M. Rocek, Nucl. Phys. B380 (1992) 128 hep-th/9112070; S.F. Hassan and A. Sen, Nucl. Phys. B405 (1993) 143 [hep-th/9210121]; M. Henningson and C. Nappi, Phys. Rev. D48 (1993) 861 hep-th/9301005; E. Kiritsis, Nucl. Phys. B405 (1993) 109 hep-th/9302033; M. Evans and I. Giannakis, Nucl. Phys. B472 (1996) 139 hep-th/9511061.

[4] X. C. de la Ossa and F. Quevedo, Nucl. Phys. B403 (1993) 377 [hep-th/9305055; A. Giveon and M. Rocek, Nucl. Phys. B421 (1994) 173 hep-th/9308154; M. Gasperini, R. Ricci and G. Veneziano, Phys. Lett. B319 (1993) 438 [hep-th/9308112]; E. Alvarez, L. Alvarez-Gaumé, J.L.F. Barbon and Y. Lozano, Nucl. Phys. B415 (1994) 71 hep-th/9309039; K. Sfetsos, Phys. Rev. D50 (1994) 2784 hep-th/9402031; E. Alvarez, L. Alvarez-Gaumé, Y. Lozano, Nucl. Phys. B424 (1994) 155 hep-th/9403155; E. Tyurin, Phys. Lett. B348 (1995) 386 hep-th/9411242; N. Mohammedi, Tours Univ. preprint [hep-th/9709071].

[5] A. Giveon, M. Porrati and E. Rabinovici, hep-th/9401139; E. Alvarez, L. AlvarezGaumé and Y. Lozano, Nucl. Phys. (Proc. Suppl.) 41 (1995) 1 hep-th/9410237.

[6] T.L. Curtright and C.K. Zachos, Phys. Rev. D49 (1994) 5408 [hep-th/9401006].

[7] E. Alvarez, L. Alvarez-Gaumé and Y. Lozano, Phys. Lett. B336 (1994) 183 hepth/9406206].

[8] Y. Lozano, Phys. Lett. B355 (1995) 165 [hep-th/9503045]; O. Alvarez and C.-Hao Liu, Commun. Math. Phys. 179 (1996) 185 hep-th/9503226].

[9] K. Sfetsos, Phys. Rev. D54 (1996) 1682 hep-th/9602179].

[10] C. Klimcik and P. Severa, Phys. Lett. B351 (1995) 455 hep-th/9502122; C. Klimcik, Nucl. Phys. (Proc. Suppl.) B46 (1996) 116 [hep-th/9509095].

[11] C. Klimcik and P. Severa, Phys. Lett. B372 (1996) 65 hep-th/9512040.

[12] K. Sfetsos, Nucl. Phys. (Proc. Suppl.) 56B (1997) 302 [hep-th/9611199].

[13] I. Bakas and K. Sfetsos, Phys. Lett. B349 (1995) 448 [hep-th/9502065; S.F. Hassan, Nucl. Phys. B460 (1996) 362 hep-th/9504148]; K. Sfetsos, Nucl. Phys. B463 (1996) 33 hep-th/9510034. 
[14] J. Polchinski, Phys. Rev. Lett. 75 (1995) 4724 hep-th/9510017.

[15] P. Horava, Phys. Lett. B231 (1989) 251; M.B. Green, Phys. Lett. B266 (1991) 325.

[16] J. Dai, R.G. Leigh and J. Polchinski, Mod. Phys. Lett. A4 (1989) 2073; R.G. Lei, Mod. Phys. Lett. A4 (1989) 2767.

[17] W. Fischler and L. Susskind, Phys. Lett. B171 (1986) 383 and B173 (1986) 262; C. Lovelace, Nucl. Phys. B273 (1986) 413; C.G. Callan, C. Lovelace, C.R. Nappi and S.A. Yost, Nucl. Phys. B288 (1987) 525.

[18] A.Yu. Alekseev and A.Z. Malkin, Commun. Math. Phys. 162 (1994) 147.

[19] E. Tyurin and R. von Unge, Phys. Lett. B382 233 hep-th/9512025.

[20] A.Yu. Alekseev, C. Klimcik and A.A. Tseytlin, Nucl. Phys. B458 (1996) 430 hepth/9509123];

[21] C. Klimcik and P. Severa, Phys. Lett. B376 (1996) 82 hep-th/9512124; Nucl. Phys. B488 (1997) 653 hep-th/9609112.

[22] C. Klimcik, IHES-P-97-58 hep-th/9707194.

[23] S.E. Parkhomenko, LANDAU-TMP-06-97 hep-th/9706199; LANDAU-TMP-09-97 hep-th/9710037.

[24] O. Alvarez, Published in Cargese 1995, Low-dimensional applications of quantum field theory, p. 1-18 hep-th/9511024.

[25] J.A. Schouten, "Ricci Calculus", (Springer, Berlin, 1954).

[26] G. Ghandour, Phys. Rev. D35 (1987) 1289; T. Curtright and G. Ghandour, "Quantum Field Theory, Statistical Mechanics, Quantum Groups and Topology", edited by T. Curtright and L. Nepomechie (World Scientific Publ. Co., Singapore, 1992).

[27] P. van Nieuwenhuizen and B. de Wit, Nucl. Phys. B312 (1989) 58.

[28] T.L. Curtright and C.K. Zachos, Phys. Rev. D52 (1995) R573 hep-th/9502126; T.L. Curtright, T. Uematsu and C. Zachos, Nucl. Phys. B469 (1996) 488 thepth/9601096]; Y. Lozano, Mod. Phys. Lett. A11 (1996) 2893 [hep-th/9610024.

[29] F. Quevedo, IFUNAM-FT-97-7 [hep-th/9706210].

[30] I. Bars and K. Sfetsos, Mod. Phys. Lett. A7 (1992) 1091 [hep-th/9110054]; E. Kiritsis and N.A. Obers, Phys. Lett. B334 (1994) 67 [hep-th/9404082]; E. Tyurin, Phys. Lett. B364 (1995) 157 hep-th/9507014. 
[31] E. Alvarez, J.L.F. Barbon and J. Borlaf, Nucl. Phys. B479 (1996) 218 hepth/9603089; H. Dorn and H.J. Otto, Phys. Lett. B381 (1996) 81 hep-th/9603186; S. Forste, A.A. Kehagias and S. Schwager, Nucl. Phys. B478 (1996) 141 hepth/9604013; J. Borlaf and Y. Lozano, Nucl. Phys. B480 (1996) 239 hep-th/9607051.

[32] A.A. Tseytlin, Mod. Phys. Lett. A6 (1991) 1721.

[33] J. Balog, P. Forgacs, Z. Horvath and L. Palla, Nucl. Phys. (Proc. Suppl.) $\underline{B 49}$ (1996) 16 [hep-th/9601091]; Phys. Lett. B388 (1996) 121 [hep-th/9606187]; L.K. Balazs and L. Palla, ITP-BUDAPEST-532 hep-th/9704137. 